\title{
Three separate regions in the prepared aniline-palladium mixture
}

\author{
Yuriy Semenyuk ${ }^{1}$, Orest Pereviznyk ${ }^{1}$, Oleksandr Reshetnyak ${ }^{1}$, Ivan Saldan ${ }^{1,2, ~ * ~}$ \\ ${ }^{1}$ Department of Physical and Colloid Chemistry, Ivan Franko National University of L'viv, 6 Kyryla and Mefodiya Str., UA-79005, L'viv, \\ Ukraine \\ ${ }^{2}$ Physics Department, Institute for Energy Technology, Kjeller, Norway
}

Email address:

semenykyu@gmail.com (Y. Semenyuk), tov.orest@gmail.com (O. Pereviznyk), reshetniak@franko.lviv.ua (O. Reshetnyak), ivan_saldan@yahoo.com (I. Saldan)

\section{To cite this article:}

Yuriy Semenyuk, Orest Pereviznyk, Oleksandr Reshetnyak, Ivan Saldan. Three Separate Regions in the Prepared Aniline-Palladium Mixture. American Journal of Physical Chemistry. Vol. 3, No. 1, 2014, pp. 1-4. doi: 10.11648/j.ajpc.20140301.11

\begin{abstract}
Polyaniline-palladium composite might be proposed for the electrode material in the oxidation of aliphatic alcohols (methanol, ethanol, ethylene glycol). Prepared mixture of aniline-palladium in sulfuric acid medium $(0<\mathrm{pH}<1)$ is investigated by SEM, PXD and FTIR. Obtained results confirm existence of three separate regions: polydisperse palladium powder with agglomerations size of $\sim 0.5$ micron in width and $\sim 3-7$ microns in length; single palladium particles $(\sim 0.5 \times 0.2$ micron) coated mainly by polyaniline form - emeraldine; aniline balls (diameter of $\sim 2-5$ microns) with $\sim 0.2$ atomic percent of $\mathrm{Pd}$ in their bulk and converted at the surface to emeraldine base and emeraldine salt.
\end{abstract}

Keywords: Aniline, Palladium, Chemical Synthesis, SEM, PXD, FTIR

\section{Introduction}

Development of metal-polymer composite has a big interest, especially for the material with high electrocatalytic properties in oxidation of aliphatic $\left(\mathrm{C}_{1}-\mathrm{C}_{2}\right)$ alcohols and their oxidized derivatives. An electrode covered by polyaniline-palladium composite is a promising object for that purpose. Therefore technology of the composite preparation acquires today a growing interest. Along with chemical procedures [1,2], electrochemical methods to synthesize palladium-filled composites are widely used and divided into two basic approaches. The first way consists in palladium reduction directly in the polymerization solution [3]. However it is complicated by the fact of palladium reduction at potentials less than $0.3 \mathrm{~V}$ while the conductivity of polyaniline in this potential region is greatly reduced. Another method is electrochemical aniline polymerization from the mixtures that contain a disperse palladium powder and might be the easiest way [4]. One of the factors responsible for the structure of such composites is the adsorption between aniline molecules and palladium particles [5]. The adsorption process occurs by the interaction between the lone electron pair of nitrogen with the $\pi$-electrons of the aromatic ring and the atoms of the metal surface. This leads to the grafting of aniline on the metal surface, thereby stabilize [6] and passivize the particle which might be bonded to a polymer during polymerization [7].

The main task of present work is detailed study of the aniline-palladium mixture. Scanning electron microscopy (SEM) observation, powder X-ray diffraction (PXD) and Fourier transform infrared (FTIR) analyses are used to investigate all initial components there.

\section{Experimental}

\subsection{Preparation of the Aniline-Palladium Mixture}

The aniline-palladium mixture was chemically prepared from disperse palladium powder. A batch of bulk palladium $(\sim 1 \mathrm{~g})$ was dissolved in a boiling solution of hydrochloric $(\sim 37 \%)$ and nitric $(\sim 68 \%)$ acids $[8,9]$ :

$$
3 \mathrm{Pd}+12 \mathrm{HCl}+2 \mathrm{HNO}_{3} \rightarrow 3 \mathrm{H}_{2}\left[\mathrm{PdCl}_{4}\right]+2 \mathrm{NO} \uparrow+4 \mathrm{H}_{2} \mathrm{O}
$$

The obtained aqueous solution of $\mathrm{H}_{2} \quad\left[\mathrm{PdCl}_{4}\right]$ was evaporated during $1 \mathrm{~h}$ to complete evolution of nitrogen (II) oxide and water. Then the dry $\mathrm{H}_{2}\left[\mathrm{PdCl}_{4}\right]$ reacted with $1 \mathrm{M}$ $\mathrm{KOH}$ aqueous solution up to $\mathrm{pH}$ value around $\sim 8$ :

$$
\mathrm{H}_{2}\left[\mathrm{PdCl}_{4}\right]+2 \mathrm{KOH} \rightarrow \mathrm{K}_{2}\left[\mathrm{PdCl}_{4}\right]+2 \mathrm{H}_{2} \mathrm{O}
$$


Palladium reduction was carried out from $\mathrm{K}_{2}\left[\mathrm{PdCl}_{4}\right]$ salt gradually adding formaldehyde till finishing gas evolution:

$$
2 \mathrm{~K}_{2}\left[\mathrm{PdCl}_{4}\right]+\mathrm{H}_{2} \mathrm{CO}+4 \mathrm{KOH} \rightarrow 2 \mathrm{Pd} \downarrow+\mathrm{CO}_{2} \uparrow+8 \mathrm{KCl}+3 \mathrm{H}_{2} \mathrm{O}
$$

Obtained solution was repeatedly washed by distilled water to extract clean palladium powder.

Commercial aniline was distilled in the chemical laboratory. The obtained aniline of $0.1 \mathrm{~mol}$ was dissolved in $1 \mathrm{M} \mathrm{H}_{2} \mathrm{SO}_{4}$ aqueous solution up to volume of 11 . After that it was $10 \mathrm{~min}$ stirred at room temperature till complete disappearing of white aniline salt deposit $(0<\mathrm{pH}<1)$ to obtain aqueous emulsion of the aniline.

Finally, to prepare the aniline-palladium mixture, $0.085 \mathrm{~g}$ of the palladium powder was added to the emulsion probe of $25 \mathrm{ml}$ followed by $\sim 2 \mathrm{~min}$ ultrasonication.

\subsection{SEM Observation}

SEM observations were performed on a Hitachi S-4800 electron microscope operated at $6.0 \mathrm{kV}$ in IFE. Some drops of the obtained mixture were deposited on a carbon adhesive tape and carbon-coated to reduce charging effects during SEM observation. SEM micrographs were taken using the backscattered electron detector to enhance the contrast between the palladium particles and aniline. Elemental analysis all of the samples was carried out by energy-dispersive X-ray spectroscopy (EDX).

\subsection{PXD Studies}

PXD experiment was carried out with the Bruker AXS D8 Advance diffractometer $\left(\mathrm{Cu} \mathrm{K} \mathrm{K}_{\alpha}\right.$ radiation, parallel beam geometry with a reflective mode, energy-dispersive detector). Data acquisition was performed in range of 10 $90^{\circ} 2 \Theta$ with a step size of $0.02^{\circ}$. The phase identification was performed by EVA program associated with PDF-4 database.

\subsection{FTIR Measurements}

FTIR spectra were taken on Bruker-ALPHA Platinum spectrometer with ATR diamond crystal accessory at room temperature in the argon filled glove-box with $\mathrm{O}_{2}$ and $\mathrm{H}_{2} \mathrm{O}$ levels less that $1 \mathrm{ppm}$. The spectra of the mixture were recorded in 4000-500 $\mathrm{cm}^{-1}$ range with resolution of $2 \mathrm{~cm}^{-1}$. Thirty two scans were averaged for background and sample spectra.

\section{Results and Discussions}

\subsection{SEM Image of the Aniline-Palladium Mixture}

For general view of the prepared mixture, SEM image in scale of 5 micron is present in Fig.1. The picture confirms a mixture of at least three different regions labeled as 1,2 and 3 , where brighter areas correspond to heavier elements in backscattering mode. Every region is analyzed by EDX spectroscopy. Atomic percent calculation is introduced in the table. Because of using aluminium sample holders small signals at Al- $K$ line are detected by EDX. Elemental analysis confirms the presence of carbon, oxygen, sulfur and palladium (hydrogen and nitrogen are not detectable by the used EDX). Among three selected areas the brightest region 1 shows the highest atomic percent of palladium. For the next regions 2 and 3 the palladium content is reduced approximately in 1 and 2 orders, respectively. EDX results show that atomic percent of carbon, oxygen and sulfur is almost the same in the different regions. This fact confirms uniform distribution of the light elements which are the components of liquid organic phase in the aniline-palladium mixture. Taking into account the EDX analysis it is suggested that region 1 might be corresponded to the palladium powder. Size of palladium agglomerations is around $\sim 0.5$ micron in width and $\sim 3-7$ microns in length with single particles approximately $\sim 0.5 \times 0.2$ micron (Fig. 1 ). Region 2 might be attributed to a round agglomeration made by these single palladium particles coated by a new organic phase since values of oxygen and sulfur are reduced while carbon increased. Dark balls in diameter of $\sim 2-5$ microns (region 3) are another organic phase with increased atomic percent of sulfur and oxygen. It might be assigned to aniline salt or its polymer form with a palladium particle inside.

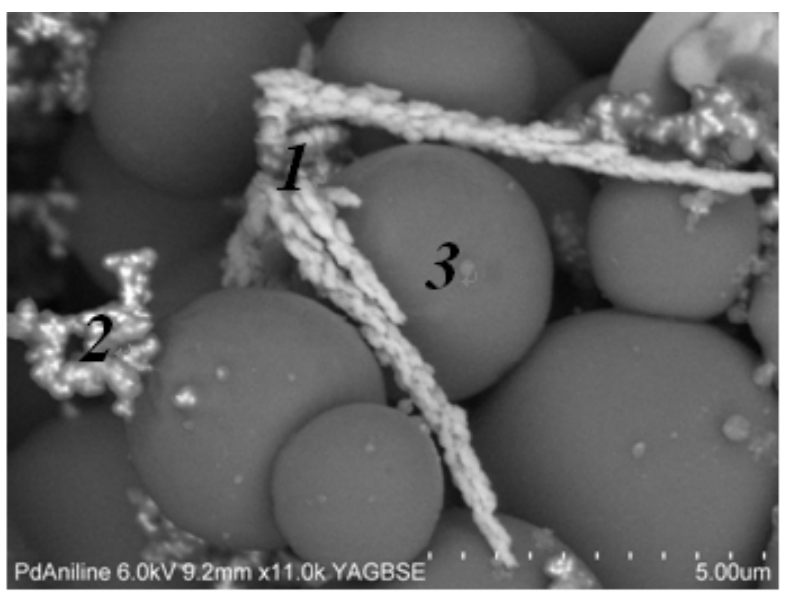

Figure 1. SEM image of the prepared aniline-palladium mixture. 1,2 and 3 are the signatures of the different regions.

Table: Atomic percent calculation of aniline-palladium mixture by EDX spectroscopy

\begin{tabular}{|c|c|c|c|c|c|c|c|c|c|c|}
\hline \multirow{2}{*}{ Region } & \multicolumn{2}{|l|}{ C-K } & \multicolumn{2}{|l|}{ O-K } & \multicolumn{2}{|l|}{$\mathrm{Al}-K$} & \multicolumn{2}{|l|}{ S-K } & \multicolumn{2}{|l|}{ Pd- $L$} \\
\hline & $\%$ & Error & $\%$ & Error & $\%$ & Error & $\%$ & Error & $\%$ & Error \\
\hline 1 & 68.09 & \pm 0.84 & 17.76 & \pm 1.02 & 0.24 & \pm 0.04 & 3.08 & \pm 0.08 & 10.84 & \pm 0.12 \\
\hline 2 & 78.14 & \pm 0.74 & 16.48 & \pm 1.03 & 0.11 & \pm 0.02 & 2.83 & \pm 0.05 & 2.44 & \pm 0.05 \\
\hline 3 & 76.65 & \pm 0.85 & 18.94 & \pm 0.89 & 0.07 & \pm 0.01 & 4.13 & \pm 0.04 & 0.21 & \pm 0.03 \\
\hline
\end{tabular}




\subsection{PDX Pattern of the Aniline-Palladium Mixture}

In Fig. 2 the PXD pattern of the prepared mixture is shown. Characteristic diffraction peaks of palladium are well visible [10] that is in agreement with EDX results in the table. Very broad feature observed in the range of $13-35^{\circ} 2 \theta$, are most likely related to the presence of the liquid organic phase in the sample. According to the $[11,12]$ this broad peak in PXD pattern might be assigned to one of the polyaniline forms.

\subsection{FTIR Spectrum of the Aniline-Palladium Mixture}

In order to understand the chemical composition of the liquid organic phase the FTIR measurements were carried out for the prepared mixture (Fig.3). Because of the liquid phase analysis, obtained infrared signals were broad. The FTIR spectrum has absorption peaks at 3340, 3210, 1630 $\mathrm{cm}^{-1}$ that confirms polyaniline form - emeraldine [13]. Both at 3340 and $3210 \mathrm{~cm}^{-1}$ corresponds to the stretching of the $\mathrm{N}-\mathrm{H}$ bonds and $1630 \mathrm{~cm}^{-1}$ of the $\mathrm{C}=\mathrm{N}$ bonds [14]. The peaks at 1410 and $1170 \mathrm{~cm}^{-1}$ correspond to $\mathrm{COOH}$ groups that might be residual formaldehyde during the mixture preparation. According to [14] these are vibration of $\mathrm{C}=\mathrm{O}$ bonds in stretching mode. Two small peaks at 2970 and 2920 $\mathrm{cm}^{-1}$ might be a signature of $\mathrm{CH}_{3}$ groups. Strong peak at $1045 \mathrm{~cm}^{-1}$ corresponds to $\mathrm{SO}_{4}$ groups [14]. The last characteristic peak at $880 \mathrm{~cm}^{-1}$ corresponds to vibration of $\mathrm{C}-\mathrm{H}$ bonds in bending mode [15]. Thus, there are no infrared signals of aniline molecules that means their polymerization to emeraldine [16,17], although in the prepared mixture there was no oxidizing agent to polymerize aniline. It is suggested that polyaniline appears during ultrasonic treatment since surface layer of aniline drops receive excess of surface energy. Complete aniline polymerization was observed by continuous $(\sim 1-2 \mathrm{~h})$ ultrasonication in [18-21]. Because of short ultrasonic treatment used for the aniline-palladium mixture, a partially aniline $\rightarrow$ polyaniline conversion is proposed in the present study.

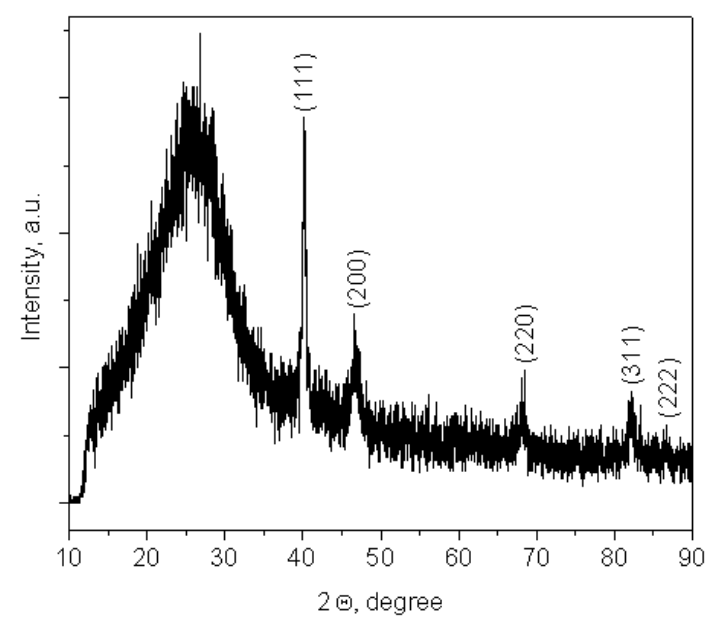

Figure 2. PXD pattern of the prepared aniline-palladium mixture.

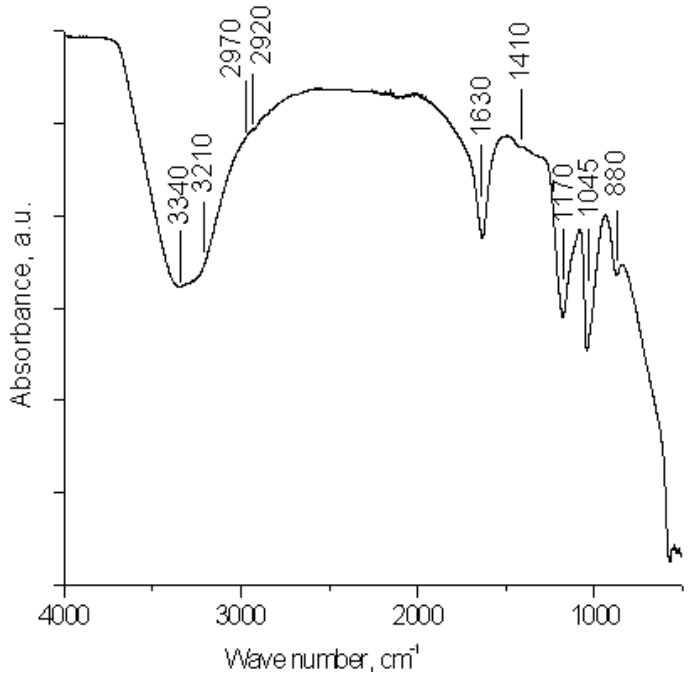

Figure 3. FTIR spectrum of the prepared aniline-palladium mixture.

Moreover the polymerization takes place mainly at the surface of aniline drops that is why they are stabilized in the mixture and introduced as ideal balls.

\section{Conclusions}

Prepared in sulfuric acid medium $(0<\mathrm{pH}<1)$ aniline-palladium mixture was studied by SEM, PXD and FTIR. Obtained results confirm existence of three separate regions: polydisperse palladium powder with agglomerations size of $\sim 0.5$ micron in width and $\sim 3-7$ microns in length; single palladium particles $(\sim 0.5 \times 0.2$ micron) coated mainly by polyaniline form - emeraldine; aniline balls (diameter of $\sim 2-5$ microns) with $\sim 0.2$ atomic percent of Pd in their bulk and converted at the surface to emeraldine base and emeraldine salt.

Recent electrochemical studies in [22] have confirmed production of the polyaniline-palladium composite from the prepared aniline-palladium mixture. The composite shows electrocatalytic properties regards to formaldehyde oxidation. However the palladium concentration on the surface of the polyaniline-palladium composite is very low and a globular morphology of the surface is observed. As a result, relatively low oxidation current is detected. It is suggested that during electrochemical polymerization of the prepared aniline-palladium mixture aniline balls become polymerized and cover the electrode surface because of the crucial adsorption factor. Therefore the small palladium concentration inside the balls does not lead to a high oxidation current.

Taking into account all experimental results it is concluded that proposed method to obtain the polyaniline-palladium composite from the prepared aniline-palladium mixture is possible. However its electrocatalytic activity is not so high since there are number of different organic phases and no Pd particles located exactly at the surface. Therefore a method of controlled nano palladium deposition on polyaniline surface might be more 
suitable to get the composite with the high electrocatalytic properties in oxidation of aliphatic $\left(\mathrm{C}_{1}-\mathrm{C}_{2}\right)$ alcohols and their oxidized derivatives.

\section{Acknowledgements}

All authors thank for instrumental assistance at Institute for Energy Technology. Dr. J.-P. Pinheiro and Dr. O. Zavorotynska are gratefully acknowledged for the SEM observation and the FTIR measurements. Dr. M.N. Guzik is thanked for guidance and test on PXD.

\section{References}

[1] L. Li, G. Yan, J. Wu, X. Yu, Q. Guo, Z. Ma, et al., "Preparation of polyaniline-palladium composite microflakes by one-step interface polymerization method", J Polym, Res, vol. 16, pp.421-426, 2009.

[2] J. E. Park, S. G. Park, A. Koukitu, O. Hatozaki, N. Oyama, "Electrochemical and chemical interactions between polyaniline and palladium nanoparticles", Synth. Met., vol. 141, pp.265-269, 2004.

[3] D. W. Hatchett, N. M. Millick, J. M. Kinyanjui, S. Pookpanratana, M. Baer, T. Hofmann, et al., "The electrochemical reduction of $\mathrm{PdCl}_{4}{ }^{2-}$ and $\mathrm{PdCl}_{6}{ }^{2-}$ in polyaniline: Influence of Pd deposit morphology on methanol oxidation in alkaline solution", Electrochim. Acta, vol. 56, pp.6060-6070, 2011.

[4] J. M. Kinyanjui, J. Hanks, D. W. Hatchett, A. Smith, M. Josowicz, "Chemical and Electrochemical Synthesis of Polyaniline/Gold Composites", J Electrochem. Soc., vol. 151, pp.D113-D120, 2004.

[5] M. G. Ramsey, G. Rosina, D. Steinmuller, H. H. Graen, F. P. Netzer, "Ordered overlayers of aniline and phenol on $\operatorname{Pd}(110)$ : Surface structure and bonding", Surf. Sci., vol. 232, pp.266-274, 1990.

[6] A. A. Athawale, S. V. Bhagwat, P. P. Katre, A. J. Chandwadkar, P. Karandikar, "Aniline as a stabilizer for metal nanoparticles", Mater. Let. Vol. 57, pp.3889-3894. 2003.

[7] U. Schmiemann, Z. Jusys, H. Baltruschat "The electrochemical stability of model inhibitors: A DEMS study on adsorbed benzene, aniline and pyridine on mono- and polycrystalline Pt, Rh and Pd electrodes", Electrochim. Acta, vol. 39, pp.561-576, 1994.

[8] G. Brauer, "Handbuch der Praparativen Anorganischen Chemie”, Moscow: Mir, 1985, vol. 5 pp. 1830-1831.

[9] R. Lidin, V. Molochko, L. Andreeva, "The Chemical Properties of Inorganic Substances", 3-rd ed., Moscow: Khimiya, 2000, pp. 444-447.
[10] C. M. Lin, T. L. Hung, Y. H. Huang, K. T. Wu, M. T. Tang, C. H. Lee, et al., "Size-dependent lattice structure of palladium studied by x-ray absorption spectroscopy", Phys. Rev. B, vol. 75, pp.125426-125432, 2007.

[11] J. P. Pouget, M. E. Jozefowicz, A. J. Epstein, X. Tang, A. G. MacDiarmid, "X-ray structure of polyaniline" Macromol., vol. 24, pp.779-789, 1991.

[12] Y. Moon, Y. Cao, P. Smith, A. J. Heeger, "X-ray diffraction of polyaniline", Polym. Commun., vol. 30, pp.196-199, 1989.

[13] A. Volkov, G. Tourillon, P. C. Lacaze, J. E. Dubois, "Electrochemical polymerization of aromatic amines: IR, XPS and PMT study of thin film formation on a Pt electrode" J Electroanal. Chem. Interfac. Electrochem., vol. 115, pp.279-291, 1980.

[14] M. Avram, G. D. Mateescu, "Spectroscopie infrarouge: applications en chimie organique", Paris: Dunod, 1970, pp. 640-642.

[15] H. Morgan, P. J. S. Foot, N. W. Brooks, "The effects of composition and processing variables on the properties of thermoplastic polyaniline blends and composites", J. Mater. Sci., vol. 36, pp.5369-5377, 2001.

[16] T. Fukuda, H. Takezoe, K. Ishikawa, A. Fukuda, H. S. Woo, S K. Jeong, et al., "Ir and Raman studies in three polyanilines with different oxidation level", Synth. Met., vol. 69, pp.175-176, 1995.

[17] S. Mu, J. Kan, "Energy density and IR spectra of polyaniline synthesized electrochemically in the solutions of strong acids", Synth. Met., vol. 98, pp.51-55, 1998.

[18] X. Jing, Y. Wang, D. Wu, L. She, Y. Guo, "Polyaniline nanofibers prepared with ultrasonic irradiation", J Polym. Sci. A: Polym. Chem., vol. 44, pp.1014-1019, 2006.

[19] H. Xia, Q. Wang, "synthesis and characterization of conductive polyaniline nanoparticles through ultrasonic assisted inverse microemulsion polymerization", J Nanopart. Res., vol. 3, pp.399-409, 2001.

[20] H. Xia, Q. Wang, "Ultrasonic Irradiation: a novel approach to prepare conductive polyaniline/nanocrystalline titanium oxide composites", Chem. Mater., vol. 14, pp.2158-2165, 2002.

[21] Y. Jiang, C. Petrier, T. D. Waite, "Kinetics and mechanisms of ultrasonic degradation of volatile chlorinated aromatics in aqueous solutions", Ultrason. Sonochem., vol. 9, pp.163-168, 2002.

[22] Yu. Semenyuk, N. Nadych, I. Saldan, O. Reshetnyak, "Formaldehyde oxidation on the electrodes modified by polyaniline/palladium-containing composite" Proceeding of the 4-th Int. Sci. Conf. Nanosize systems: structure, properties, technologies. Ukraine. p. 406, 2013. 